\title{
Using Probabilistic Model Checking for Dynamic Power Management
}

\author{
Gethin Norman ${ }^{1}$, David Parker ${ }^{1}$, Marta Kwiatkowska ${ }^{1}$, Sandeep Shukla ${ }^{2}$ and Rajesh Gupta ${ }^{3}$ \\ ${ }^{1}$ School of Computer Science, University of Birmingham, Birmingham, UK \\ ${ }^{2}$ Bradley Department of Electrical \& Computer Engineering, Virginia Tech, Blacksburg, USA \\ ${ }^{3}$ Department of Information \& Computer Science, University of California, San Diego, USA
}

\begin{abstract}
Dynamic power management (DPM) refers to the use of runtime strategies in order to achieve a tradeoff between the performance and power consumption of a system and its components. We present an approach to analysing stochastic DPM strategies using probabilistic model checking as the formal framework. This is a novel application of probabilistic model checking to the area of system design. This approach allows us to obtain performance measures of strategies by automated analytical means without expensive simulations. Moreover, one can formally establish various probabilistically quantified properties pertaining to buffer sizes, delays, energy usage etc., for each derived strategy.
\end{abstract}

Keywords: Power management, formal methods, embedded systems, model checking, probabilistic model checking.

\section{Introduction}

The nature of computing has been changing over the last few years from server, workstation and desktop based computing to embedded, ubiquitous and pervasive computing. Handheld devices, wireless sensors and biomedical devices are gaining more and more prominence in all arenas of human life. However, as we move from the wired to wireless domain, power savings in these computing devices become more crucial. As a result, much research has been done in the area of low-power design, power management and the balance between computation and communication power. Each kind of approach to power savings has its own limitations. For example, circuit-level or architecture-level mechanisms cannot take advantage of application characteristics. As a result, system-level power management, which is characterised by operating system controlled power saving measures based on the observation of application characteristics, has gained significant attention in the last few years. There are two distinct flavours of system-level power management: dynamic voltage/frequency scaling (DVS/DFS) and dynamic power management (DPM). In this paper, we focus on the latter.

Dynamic power management (DPM) is a way to save energy in devices which, under operating system control, can be switched either on and off or between several power states of varying power consumption.

Correspondence and offprint requests to: Gethin Norman, School of Computer Science, University of Birmingham, Birmingham B15 2TT, United Kingdom. E-mail: G.Norman@cs.bham.ac.uk 
DPM has gained considerable attention over the last few years, a trend evidenced in the research literature HAW96, SCB96, BM98, BMM01, SG01, RIG00, CBBM99, ISG02, as well as concerted industry efforts such as Microsoft's OnNow [Mic98] and ACPI [ACP. Due to the importance of the minimising power consumption in today's embedded systems, a lot of work has been initiated in both the component manufacturing industry and the systems design industry.

A survey of most of the techniques developed for DPM before 2000 can be found in BBM00. In this extensive review, the approaches to DPM are classified into predictive schemes and stochastic optimum control schemes. Predictive schemes attempt to predict a device's usage behaviour in the future, typically based on the past history of usage patterns, and decide to change power states of the device accordingly. Stochastic approaches make probabilistic assumptions (based on observations) about usage patterns and exploit the nature of the probability distribution to formulate an optimisation problem, the solution to which drives the DPM strategy.

It has been noted that predictive schemes are mostly based on devices with two power-saving states, whereas there are many instances of devices in the embedded world which have more than two states. Examples of such devices may be found in BBM00, SBM99. In order to provide DPM strategies for multi-state systems, the stochastic optimum control approach has been proposed in the literature BBM00, SBM99. BBPM99, CBBM99, QP99, QWP01. However, such stochastic approaches also have their drawbacks, including the fact that they make many assumptions about the probabilistic nature of the inputs and may be more computationally expensive to implement.

Much of the previous work on dynamic power management has been based on ad-hoc techniques, such as the use of regression equations, interpolation or learning based methods. Stochastic approaches tend to be more formal in the sense that they are based on mathematical models which make precise assumptions about the probabilistic characteristics of, for example, when service requests arrive at a device and how long the device takes to respond to these requests. Validation and analysis of the stochastic DPM schemes, however, is less formal, evaluation usually being carried out with simulation techniques which are time consuming and often not completely reliable.

In this paper, we illustrate the applicability of probabilistic model checking, an automatic formal verification technique for the analysis of systems which exhibit probabilistic behaviour, to the area of dynamic power management. We show how the probabilistic model checking tool PRISM [KNP04, Pri] can be used to automatically provide a detailed comprehensive of stochastic DPM schemes. Furthermore, this analysis is more accurate than that obtained by simulation which typically yields only average case behaviour. An earlier version of this work appeared in $\left[\mathrm{NPK}^{+} 02, \mathrm{NPK}^{+} 03\right]$.

\subsection{Organisation}

Section 2 introduces the stochastic approach to DPM, with references to the existing literature. Section 3 describes the basics of probabilistic model checking and the PRISM tool. Section 4 shows in detail how probabilistic model checking and PRISM have been applied to the analysis of DPM strategies and together with MAPLE Map used to generate DPM strategies. Finally, Section 5 concludes the paper.

\section{Stochastic Approaches to DPM}

The stochastic version of the DPM problem basically requires one to devise a strategy (policy) which may be probabilistic, in the sense that the actions to be taken by the strategy may have probabilities attached to them. Unlike deterministic strategies, where a particular state of the system will lead the strategy to take a deterministic action, here the strategy can choose between multiple actions with pre-designated probabilities.

In recent years, several approaches for designing stochastic DPM strategies have been proposed PBBM98 BBPM99, BBM00, CBBM99, QP99, QWP00, QWP01, SBM99. These methodologies are based on a stochastic model of the DPM problem, which incorporates the probabilistic characteristics of request arrivals to the device, the device response time distribution, the power consumption by the device in various states and the energy consumption when the device changes state. From this stochastic model, an exact optimisation problem is formulated, the solution to which is the required optimal stochastic DPM policy. The strategy devised must ensure that power savings are not achieved at an undue cost in performance. One approach, for example, is to construct a policy which optimises the average energy usage while bounding average delay. 
The constructed policies are usually validated by simulation to check for the soundness of the modelling assumptions, and the effectiveness of the strategies in practice QP99, PBBM98.

The stochastic models which have been used in the literature are discrete-time Markov chains PBBM98 BBPM99, continuous-time Markov chains QP99, QWP00, QWP01 and their variants SBM99. The approaches vary in the modelling of time: in the continuous-time case, mode switching commands can be issued at any time, and events can happen at any time. In the discrete-time case, all events and actions occur at certain discrete time points. In practice, such stochastic modelling seems to work well for specific kinds of applications. Generally, the stochastic matrices for these models are created manually. In QWP00, stochastic Petri nets are used, which allows automatic generation of the stochastic matrices and formulation of the optimisation problems.

\section{Probabilistic Model Checking}

Model checking is a well established and successful technique for the automatic verification of finite state systems. In recent years, a significant amount of work has gone into probabilistic model checking, which allows for verification of systems that exhibit probabilistic behaviour. These include randomised algorithms, which use probabilistic choices or electronic coin flipping, and unreliable or unpredictable processes, such as fault-tolerant systems or communication networks.

To perform probabilistic model checking one first constructs a probabilistic model of the system under study. As in the non-probabilistic case, this model is usually a labelled transition system which defines the set of all possible states that the system can be in and the transitions which can occur between these states. However, in this case, one must also augment the model with information about the likelihood that each transition will take place.

Properties of the system which are to be verified are then specified, typically in probabilistic extensions of temporal logic. These allow specification of properties such as: "shutdown occurs with probability at most 0.01 "; or "the video frame will be delivered within $5 \mathrm{~ms}$ with probability at least 0.97 ". A probabilistic model checker applies algorithmic techniques to analyse the state space of the probabilistic model and determine whether these specifications are satisfied. Typically, this involves computation of one or more probabilities or performance measures. The operations required are graph-based analysis and solution of linear equation systems or linear optimisation problems.

\subsection{Probabilistic Models}

Models used in probabilistic model checking are commonly variants of Markov chains. The simplest is discretetime Markov chains (DTMCs). A DTMC is defined by a set of states $S$ and a probability transition matrix $\mathbf{P}: S \times S \rightarrow[0,1]$, where $\sum_{s^{\prime} \in S} \mathbf{P}\left(s, s^{\prime}\right)=1$ for all $s \in S$. This gives the probability $\mathbf{P}\left(s, s^{\prime}\right)$ that a transition will take place from state $s$ to state $s^{\prime}$.

Continuous-time Markov chains (CTMCs) extend DTMCs by allowing transitions to occur in real-time, rather than only in discrete steps. A CTMC is defined by a set of states $S$ and a transition rate matrix $\mathbf{R}: S \times S \rightarrow \mathbb{R}_{\geqslant 0}$. The rate $\mathbf{R}\left(s, s^{\prime}\right)$ defines the delay before which a transition between states $s$ and $s^{\prime}$ is enabled. The delay is sampled from a negative exponential distribution with parameter equal to this rate, i.e. the probability of the transition being enabled within $t$ time units is $1-e^{-\mathbf{R}\left(s, s^{\prime}\right) \cdot t}$. When $\mathbf{R}\left(s, s^{\prime}\right)>0$ for two target states, a race occurs and the transition which becomes enabled first is the one taken. Exponentially distributed delays are often suitable for modelling component lifetimes and inter-arrival times. They can also be used to approximately model more complex probability distributions.

\subsection{Analysis of Probabilistic Models}

Similarly to the conventional, non-probabilistic case, probabilistic model checking usually constitutes verifying whether or not some temporal logic formula is satisfied by a model. The two most common temporal logics for this purpose are PCTL HJ94, BdA95] and CSL [ASSB96, BKH99, both extensions of the logic CTL. PCTL is used to specify properties for DTMCs and MDPs; CSL is used for CTMCs.

One common feature of the two logics is the probabilistic operator $\mathcal{P}$, which allows one to reason about the 
probability that executions of the system satisfy some property. For example, the formula $\mathcal{P}_{\geqslant 1}[\diamond$ terminate $]$ states that, with probability 1 , the system will eventually terminate. On the other hand, the formula $\mathcal{P}_{\geqslant 0.95}[\neg$ repair $\mathcal{U} \leqslant 200$ terminate $]$ asserts that, with probability 0.95 or greater, the system will terminate within 200 time units and without requiring any repairs. These properties can be seen as analogues of the non-probabilistic case, where a formula would typically state that all executions satisfy a particular property, or that there exists an execution which satisfies it. CSL also provides the $\mathcal{S}$ operator to reason about steadystate (long-run) behaviour. The formula $\mathcal{S}_{<0.01}\left[q u e u e \_s i z e=\max \right]$, for example, states that, in the long-run, the probability that a queue is full is less than 0.01 .

Strictly speaking, probabilistic specifications in PCTL and CSL (such as the examples above) always contain a probability bound, so that properties are either true or false for a given system. In practice, however, this can be relaxed. Model checking algorithms for PCTL and CSL typically proceed by computing the actual probability and then comparing it to the bound. Hence, in practice, we can write an expression of the form $\mathcal{P}_{=\text {? }}[\nabla$ terminate], for which the model checker will return the actual probability that the system terminates. In many cases, the most useful form of analysis is to compute such values for a range of models or properties. For example, one might determine $\mathcal{P}=$ ? $\left[\diamond^{\leqslant t}\right.$ terminate $]$ for a range of values of $t$ in order to gain insight into the likelihood of the system terminating as time progresses.

Further properties can be analysed by introducing the notion of costs (or, conversely, rewards). If each state of the probabilistic model is assigned a real-valued cost, we can compute properties such as the expected cost to reach a certain states, the expected accumulated cost over some time period, or the expected cost at a particular time instant. As in the previous paragraph, such properties can also be expressed concisely and unambiguously in temporal logic [dA97, BHHK00].

\subsection{PRISM: A Probabilistic Model Checker}

PRISM KNP04, Pri is a probabilistic model checker developed at the University of Birmingham. It supports analysis of the two types of probabilistic models discussed previously: discrete-time Markov chains (DTMCs) and continuous-time Markov chains (CTMCs), and also Markov decision processes (MDPs) which we do not use here. It verifies properties specified in the temporal logics PCTL (for DTMCs and MDPs) and CSL (for CTMCs). Other probabilistic model checkers include ProbVerus HGCC99 for DTMCs, EトMC ${ }^{2}$ HKMKS00] for CTMCs and DTMCs, and RAPTURE [JDL02 for MDPs.

PRISM has been used to analyse a wide range of case studies, including probabilistic algorithms for problems such as anonymity, contract signing, leader election and consensus; and performance analysis of various queueing systems, communication networks and manufacturing systems. See [Pri] for further details. Figure 1 shows a screenshot of the tool running.

Probabilistic models to be analysed in PRISM are specified in the PRISM language, which is based on the Reactive Modules formalism of Alur and Henzinger AH99. The basic components of this language are modules and variables. A system is constructed as the parallel composition of a set of modules. A module contains a number of variables which express the state of the module. Its behaviour is given by a set of guarded commands of the form:

\footnotetext{
[] <guard $>\rightarrow$ <command $>$;
}

The guard is a predicate over all the variables of the system and the command describes a transition which the module can make if the guard is true. A command is specified by defining the new values of the variables of that module. This means that a module can read all of the variables in the system but only write to its own local variables. In general, the behaviour of a module is probabilistic, in which case a command takes the form:

$$
\langle\text { prob> : <action> }+\cdots+\langle\text { prob> : <action> }
$$

where $\langle$ prob $\rangle$ is a probability when the model is a DTMC or MDP and a non-negative, real value (taken to be the parameter of an exponential distribution) when it is a CTMC. In addition, the pair of square brackets at the start of a guarded command can contain a label. Actions from different modules with the same label take place synchronously. See Pri, Par02 for more details.

The overall functionality of the PRISM tool is as follows. First, it reads and parses a model description in the PRISM language. It then constructs the corresponding DTMC, CTMC or MDP, computes the set of all reachable states, and identifies any deadlock states (i.e. reachable states with no outgoing transitions). 


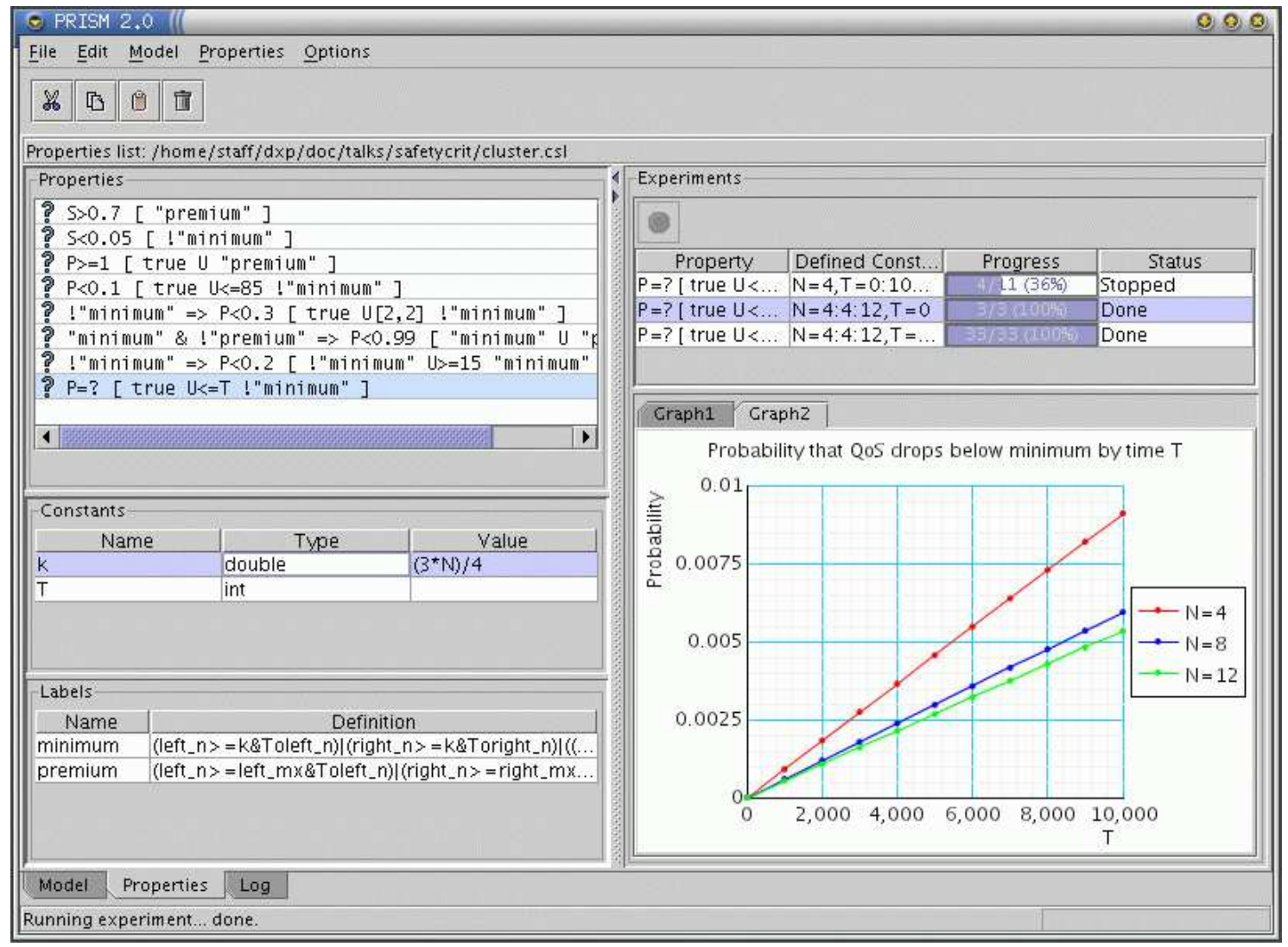

Fig. 1. Screenshot of the PRISM graphical user interface

If required, the transition matrix of the probabilistic model constructed can be exported for use in another tool. Typically, though, PRISM then parses one or more properties in PCTL or CSL and performs model checking, determining whether the model satisfies each property. A prototype version of PRISM has also been developed which supports model checking of cost and reward related properties, as described in the previous section.

\section{Probabilistic Model Checking and DPM}

In this section, we describe how probabilistic model checking and, in particular, PRISM can be applied to the analysis of DPM strategies obtained using the approaches of QP99. PBBM98. These approaches are based on constructing a probabilistic model of the dynamic power management system from which, for a given constraint, an optimisation problem is constructed. The solution to this problem is the optimum randomised power management strategy satisfying this constraint.

We show how PRISM can be used to construct a probabilistic model of dynamic power management. The corresponding optimisation problem (as described in QP99, PBBM98) is then solved with the symbolic solver MAPLE Map. Finally, we again use PRISM to automatically validate and analyse the derived policies. Note that we use MAPLE to solve the optimisation problem because PRISM does not currently support methods for solving problems of this type ${ }^{1}$

1 PRISM does support the solution of the optimisation problems generated when verifying MDPs. However, these problems are specific instances of the Stochastic Shortest Path Problem [BT91, Ber95] and, since the solution techniques employed by PRISM rely on this fact, these methods cannot be applied to general optimisation problems. 
Table 1. Average power consumption (W) and service times (ms) for each power state

\begin{tabular}{lccccc}
\hline & sleep & standby & idlelp & idle & active \\
\hline power $(\mathrm{W})$ & 0.1 & 0.3 & 0.8 & 1.5 & 2.5 \\
service time $(\mathrm{ms})$ & 0 & 0 & 0 & 0 & 1 \\
\hline
\end{tabular}

Table 2. Average transition times (ms) between power states

\begin{tabular}{lccccc}
\hline & active & idle & idlelp & standby & sleep \\
\hline active & - & 1 & 5 & 220 & 600 \\
idle & 1 & - & 5 & 220 & 600 \\
idlelp & 5 & - & - & 220 & 600 \\
standby & 220 & - & - & - & 600 \\
sleep & 600 & - & - & - & - \\
\hline
\end{tabular}

This approach differs from the previously employed techniques for the validation and analysis of DPM strategies, which rely on simulation or the actual implementation of the schemes in device drivers. The advantage of the probabilistic model checking approach is that it avoids the higher cost of simulation and benefits of detailed analysis before deployment in hardware. Furthermore, the analysis is more accurate than that obtained by simulation which typically yields only average case behaviour.

\subsection{Modelling DPM in PRISM}

We have applied probabilistic model checking to two stochastic DPM approaches: that of Benini et al. PBBM98, BBPM99, based on discrete-time Markov chains, and that of Qiu et al. QP99, QWP00, QWP01, based on continuous-time Markov chains. In this section we describe the DTMC approach in detail.

The approach is described through the example of [PBBM98, BBPM99], an IBM TravelStar VP diskdrive [BM]. The device has 5 power states, labelled sleep, standby, idle, idlelp and active. It is only in the state active that the drive can perform data read and write operations. In state $i d l e$, the disk is spinning while some of the electronic components of the disk drive have been switched off. The state idlelp (idle low power) is similar except that it has a lower power dissipation. The states standby and sleep correspond to the disk being spun down. Tables 1 and 2 show actual data for these power states. Table 1 gives the average power consumption $(\mathrm{W})$ and the service time $(\mathrm{ms})$ for each state. Table 2 shows the average time (ms) to transition between each pair of states.

We now describe how the system is modelled in the PRISM language. Following the approach of PBBM98, BBPM99, the model constructed is a discrete-time Markov chain (DTMC). Based on the fastest possible transition performed by system, we choose a time resolution of $1 \mathrm{~ms}$ for the model, i.e. each discrete-time step of the DTMC will correspond to $1 \mathrm{~ms}$.

The basic structure of the DPM model can be seen in Figure 2. The model consists of: a Service Provider (SP), which represents the device under power management control; a Service Requester (SR), which issues requests to the device; a Service Request Queue (SRQ), which stores requests that are not serviced immediately; and the Power Manager (PM), which issues commands to the SP, based on observations of the system and a stochastic DPM policy. Each component is represented by an individual PRISM module, which we now consider in turn.

\subsubsection{Modelling the power manager (PM).}

The PM decides to which state the SP should move at each time step. To model this, we split each step into two parts: in the first, the PM (instantaneously) decides what the SP should do next (based on the current state); and in the second, the system makes a transition (with the SP's move based on the choice made by the PM). To achieve this, we introduce the CLOCK module, given in Figure 3 . Transitions of this module are labelled alternately with tick and tock. The PM is then constructed to synchronise with the CLOCK on tick, while the remaining components are constructed to synchronise with the CLOCK on tock. A generic PM has the form given in Figure 4. For example, if the state of the system satisfies cond ${ }_{1}$, then the PM 


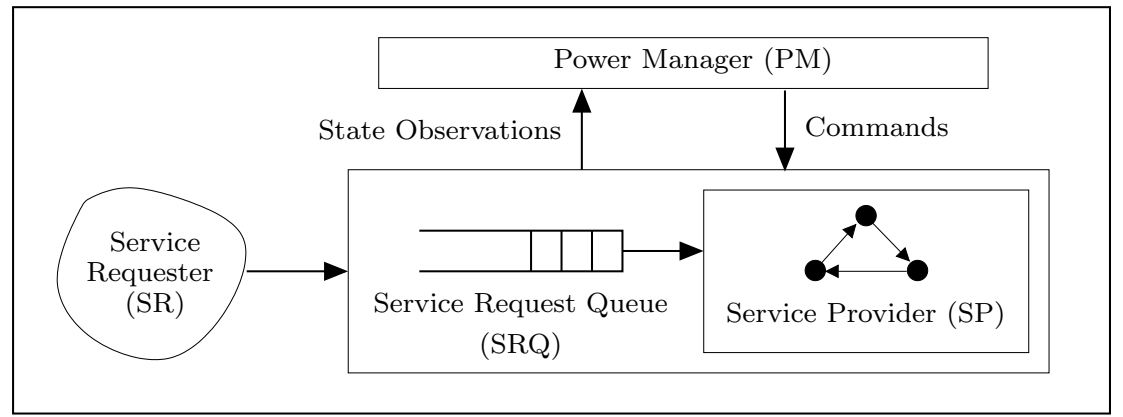

Fig. 2. The System Model

module CLOCK

c : $[0 . .1]$ init 0 ;

$\left[\right.$ tick] $\mathrm{c}=0 \rightarrow\left(\mathrm{c}^{\prime}=1\right)$

[tock] $\mathrm{c}=1 \rightarrow\left(\mathrm{c}^{\prime}=0\right)$

endmodule

Fig. 3. PRISM module for the clock

decides that with probability prob_active ${ }_{1}$ the $\mathrm{SP}$ will move to active, with probability prob_idle ${ }_{1}$ the SP will move to idle, with prob_idlelp ${ }_{1}$ to idlelp, prob_standby ${ }_{1}$ to standby, and prob_sleep ${ }_{1}$ to sleep.

\subsubsection{Modelling the service provider (SP).}

As mentioned above, the SP (the disk drive) has 5 power states (active, idle, idlelp, standby and sleep). These states and the possible transitions between them are shown in Table 2. The actual PRISM code is shown in Figure 5. Recall that the SP synchronises with the clock on tock. Hence, all of its guarded commands are labelled with this action. Note also that the behaviour of the SP depends on the PM, so the guards reference the variable $\mathrm{pm}$.

Since a time resolution of $1 \mathrm{~ms}$ has been chosen, in order to correctly model transitions with delays longer than this time resolution transient states are introduced. For example, the transient state active_idlelp is used to model the non-unitary time transition from active to idlelp. The transition probabilities in the transient

module PM

$\mathrm{pm}:[0 . .4] ; / / 0$ - go to active, 1 - go to idle, 2 - go to idlelp, 3 - go to standby, 4 - go to sleep

$[$ tick $] \operatorname{cond}_{1} \rightarrow$ prob_active $_{1}:\left(\mathrm{pm}^{\prime}=0\right)$

+ prob_idle $1:\left(\mathrm{pm}^{\prime}=1\right)$

+ prob_idlelp $1:\left(\mathrm{pm}^{\prime}=2\right)$

+ prob_standby $_{1}:\left(\mathrm{pm}^{\prime}=3\right)$

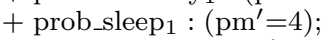

[tick] cond $_{2} \rightarrow$ prob_active $_{2}:\left(\mathrm{pm}^{\prime}=0\right)$

+ prob_idle $2:\left(\mathrm{pm}^{\prime}=1\right)$

+ prob_idlelp $_{2}:\left(\mathrm{pm}^{\prime}=2\right)$

+ prob_standby $2:\left(\mathrm{pm}^{\prime}=3\right)$

+ prob_sleep $2:\left(\mathrm{pm}^{\prime}=4\right)$;

Fig. 4. PRISM module for the power manager 
module SP

sp : [0..10] init 9;

$/ / 0=$ active, $1=i d l e, 2=$ active_idlelp $, 3=i d l e l p, 4=i d l e l p \_a c t i v e, 5=$ active_standby

$/ / 6=$ standby, $7=$ standby_active, $8=$ active_sleep, $9=$ sleep, $10=$ sleep_active

// states where PM has no control (transient states)

[tock] $\mathrm{sp}=2 \rightarrow 0.75:\left(\mathrm{sp}^{\prime}=\mathrm{sp}\right)+0.25:\left(\mathrm{sp}^{\prime}=3\right)$;

[tock] $\mathrm{sp}=4 \rightarrow 0.75:\left(\mathrm{sp}^{\prime}=\mathrm{sp}\right)+0.25:\left(\mathrm{sp}^{\prime}=0\right) ;$

tock] $\mathrm{sp}=5 \rightarrow 0.995:\left(\mathrm{sp}^{\prime}=\mathrm{sp}\right)+0.005:\left(\mathrm{sp}^{\prime}=6\right)$

[tock] $\mathrm{sp}=7 \rightarrow 0.995:\left(\mathrm{sp}^{\prime}=\mathrm{sp}\right)+0.005:\left(\mathrm{sp}^{\prime}=0\right)$

tock] $\mathrm{sp}=8 \rightarrow 0.9983:\left(\mathrm{sp}^{\prime}=\mathrm{sp}\right)+0.0017:\left(\mathrm{sp}^{\prime}=9\right)$

[tock] $\mathrm{sp}=10 \rightarrow 0.9983:\left(\mathrm{sp}^{\prime}=\mathrm{sp}\right)+0.0017:\left(\mathrm{sp}^{\prime}=0\right)$;

// PM: goto active

[tock] $\mathrm{pm}=0 \wedge(\mathrm{sp}=0 \vee \mathrm{sp}=1) \rightarrow\left(\mathrm{sp}^{\prime}=0\right) ;$

[tock] $\mathrm{pm}=0 \wedge \mathrm{sp}=3 \rightarrow\left(\mathrm{sp}^{\prime}=4\right)$;

[tock] $\mathrm{pm}=0 \wedge \mathrm{sp}=6 \rightarrow\left(\mathrm{sp}^{\prime}=7\right)$;

[tock] $\mathrm{pm}=0 \wedge \mathrm{sp}=9 \rightarrow\left(\mathrm{sp}^{\prime}=10\right)$;

// PM: goto idle

[tock] $\mathrm{pm}=1 \wedge(\mathrm{sp}=0 \vee \mathrm{sp}=1) \rightarrow\left(\mathrm{sp}^{\prime}=1\right) ;$

[tock] $\mathrm{pm}=1 \wedge(\mathrm{sp}=3 \vee \mathrm{sp}=6 \vee \mathrm{sp}=9) \rightarrow\left(\mathrm{sp}^{\prime}=\mathrm{sp}\right)$

// PM: goto idlelp

[tock] $\mathrm{pm}=2 \wedge(\mathrm{sp}=0 \vee \mathrm{sp}=1) \rightarrow\left(\mathrm{sp}^{\prime}=2\right) ;$

[tock] $\mathrm{pm}=2 \wedge \mathrm{sp}=3 \rightarrow\left(\mathrm{sp}^{\prime}=\mathrm{sp}\right)$;

// PM: goto standby

[tock] $\mathrm{pm}=3 \wedge(\mathrm{sp}=0 \vee \mathrm{sp}=1 \vee \mathrm{sp}=3) \rightarrow\left(\mathrm{sp}^{\prime}=5\right) ;$

[tock] $\mathrm{pm}=3 \wedge \mathrm{sp}=6 \rightarrow\left(\mathrm{sp}^{\prime}=\mathrm{sp}\right)$;

// PM: goto sleep

[tock] $\mathrm{pm}=4 \wedge(\mathrm{sp}=0 \vee \mathrm{sp}=1 \vee \mathrm{sp}=3 \vee \mathrm{sp}=6) \rightarrow\left(\mathrm{sp}^{\prime}=8\right) ;$

[tock] $\mathrm{pm}=4 \wedge \mathrm{sp}=9 \rightarrow\left(\mathrm{sp}^{\prime}=9\right)$;

endmodule

Fig. 5. PRISM module for the service provider

module SR

sr : [0..1] init $0 ; / / 0-$ idle and 1 - req

[tock] $\mathrm{sr}=0 \rightarrow 0.898:\left(\mathrm{sr}^{\prime}=0\right)+0.102:\left(\mathrm{sr}^{\prime}=1\right)$

[tock] $\mathrm{sr}=1 \rightarrow 0.454:\left(\mathrm{sr}^{\prime}=0\right)+0.546:\left(\mathrm{sr}^{\prime}=1\right)$

endmodule

Fig. 6. PRISM module for the service requester

states, taken directly from the data of PBBM98, BBPM99, are chosen such that the mean times to move between power states are as given in Table 2. Note that we suppose that the power dissipation in these transient states is high $(2.5 \mathrm{~W})$.

\subsubsection{Modelling the service requester (SR) and queue (SRQ).}

Similarly to the SP, both the SR and the SRQ synchronise with the clock on tock. The SR has two states: idle where no requests are generated and req where one request is generated per time step (1ms). The probabilities associated with the transitions between these states are based on time-stamped traces of disk access measured on real machines BBPM99. The module for the SR is given in Figure 6.

The SRQ models a queue of service requests. It responds to the arrival of requests from the SR and the service of requests by the SP. The queue size will only decrease when the SR and SP are in states idle and active, respectively. On the other hand, it will only increase when the SR is in state req and the SP is not active. The PRISM code is given in Figure 7. 
const QMAX $=2 ; / /$ maximum size of the queue

module SRQ

$\mathrm{q}:[0 . . \mathrm{QMAX}]$ init $0 ; / /$ size of queue

// $S P$ is active

[tock] $\mathrm{sr}=0 \wedge \mathrm{sp}=0 \rightarrow\left(\mathrm{q}^{\prime}=\max (\mathrm{q}-1,0)\right)$;

[tock] $\mathrm{sr}=1 \wedge \mathrm{sp}=0 \rightarrow\left(\mathrm{q}^{\prime}=\mathrm{q}\right)$;

// $S P$ is not active

[tock] $\mathrm{sr}=0 \wedge \mathrm{sp}>0 \rightarrow\left(\mathrm{q}^{\prime}=\mathrm{q}\right)$;

[tock] $\mathrm{sr}=1 \wedge \mathrm{sp}>0 \rightarrow\left(\mathrm{q}^{\prime}=\min (\mathrm{q}+1, \mathrm{QMAX})\right)$;

endmodule

Fig. 7. PRISM module for the service request queue

module BATTERY

bat : [0..1] init 1;// 0 - battery off and 1 - battery on

[tock] bat $=1 \rightarrow 0.999999:\left(\mathrm{bat}^{\prime}=1\right)+0.000001:\left(\mathrm{bat}^{\prime}=0\right)$;

endmodule

Fig. 8. PRISM module for the battery

\subsubsection{Modelling a Finite Time Horizon.}

We suppose that there is a time horizon of one million time steps. To model this horizon, an additional module representing a battery with an expected life span of 1 million time steps is added (see Figure 8). Note that, once the battery reaches state 0 , it cannot perform the action tock which prevents any other modules in the system from performing this action. Hence, the rest of the system can no longer continue (i.e. the states where bat $=0$ act as sink states).

\subsection{Policy Construction}

Using the PRISM language description detailed in the previous sections, the PRISM model checking tool can be used to construct a generic model of the power management system. From the transition matrix of this system, the linear optimisation problem whose solution is the optimal policy can be formulated, as described in PBBM98, BBPM99. This optimisation problem is then passed to the MAPLE symbolic solver. Table 3 shows policies constructed in this way for a range of constraints on the average size of the service request queue. The first column lists the constraint; the second column summarises the corresponding policy.

\subsection{Policy Analysis}

Once a policy has been constructed, its performance can be automatically analysed through probabilistic model checking, as described in Section3. The generic power manager PRISM module is modified to represent a specific policy. Figure9 9 shows an example of this for the constraint "queue size is less than 0.05 ". This can be seen to correspond to the policy in the 5th row of the table in Table 3 . PRISM is then used to construct and analyse the DTMC for this policy.

We now present a representative set of results obtained through probabilistic model checking that demonstrate the utility and power of this approach. The policies analysed are those constructed from a range of constraints on the average queue length. In Table 4, the following properties have been computed: "average power consumption", "average queue size" and "average number of lost requests". Using PRISM, we asso- 
Table 3. Optimum policies under varying constraints on the average queue size

\begin{tabular}{|c|c|}
\hline constraint & optimum policy \\
\hline$\leqslant 1.5$ & $\begin{array}{l}\text { if the SP is active and the SRQ is not full then } \\
\text { with probability } 1 \text { goto idle } \\
\text { elseif the SR is idle, the SP is in sleep and the SRQ is full then } \\
\text { with probability } 0.00000047 \text { goto active } \\
\text { elseif the SR is in req and the SP is idle then } \\
\text { with probability } 1 \text { goto active } \\
\text { end }\end{array}$ \\
\hline$\leqslant 1$ & $\begin{array}{l}\text { if the SP is active and the SRQ is not full then } \\
\text { with probability } 1 \text { goto idle } \\
\text { elseif the SR is idle, the SP is in sleep and the SRQ is full then } \\
\text { with probability } 0.00000150 \text { goto active } \\
\text { elseif the SR is in req and the SP is idle then } \\
\text { with probability } 1 \text { goto active } \\
\text { end }\end{array}$ \\
\hline$\leqslant 0.5$ & $\begin{array}{l}\text { if the SP is active and the SRQ is not full then } \\
\text { with probability } 1 \text { goto idle } \\
\text { elseif the SR is idle, the SP is in sleep and the SRQ is full then } \\
\text { with probability } 0.00000582 \text { goto active } \\
\text { elseif the SR is in req and the SP is idle then } \\
\text { with probability } 1 \text { goto active } \\
\text { end }\end{array}$ \\
\hline$\leqslant 0.1$ & $\begin{array}{l}\text { if the SP is active and the SRQ is not full then } \\
\text { with probability } 1 \text { goto idle } \\
\text { elseif the SR is idle and the SP is idle then } \\
\text { with probability } 0.95197200 \text { goto active } \\
\text { elseif the SR is in req and the SP is idle then } \\
\text { with probability } 1 \text { goto active } \\
\text { elseif the SP is in sleep then } \\
\text { with probability } 1 \text { goto active } \\
\text { end }\end{array}$ \\
\hline$\leqslant 0.05$ & $\begin{array}{l}\text { if the SP is active, the SR is idle and the SRQ is empty then } \\
\text { with probability } 0.36316067 \text { goto idle } \\
\text { elseif the SP is idle then } \\
\text { with probability } 1 \text { goto active } \\
\text { elseif the SP is in sleep then } \\
\text { with probability } 1 \text { goto active } \\
\text { end }\end{array}$ \\
\hline$\leqslant 0.001$ & $\begin{array}{l}\text { if the SP is active, the SR is idle and the SRQ is empty then } \\
\text { with probability } 0.05068717 \text { goto idle } \\
\text { elseif the SP is idle then } \\
\text { with probability } 1 \text { goto active } \\
\text { elseif the SP is in sleep then } \\
\text { with probability } 1 \text { goto active } \\
\text { end }\end{array}$ \\
\hline
\end{tabular}

ciate a cost with each state and then compute the expected accumulated cost of the system until it reaches a state where the battery has run out. For example, to determine the average power consumption, the cost associated with each state is determined by the state of the SP and the data given in Table 1.

From the table, we can see that the average power consumption of a policy decreases as the constraint on queue size is relaxed (i.e. the requested average queue size is increased). We can also validate the policy by confirming that the expected size of the queue matches the value in the constraint which was used to construct it. Finally, we see that a side-effect of this is that the average number of requests lost also increases.

In Figure 10, we show graphical results for a range of policies. Using the same assignments of model states to costs as discussed above, we (automatically in PRISM) compute and plot, for a range of values of $T$ : "expected power consumption by time $T$ ", "expected queue size at time $T$ ", and "expected number of lost requests by time $T$ ". The first and third properties are determined by computing expected cost cumulated 


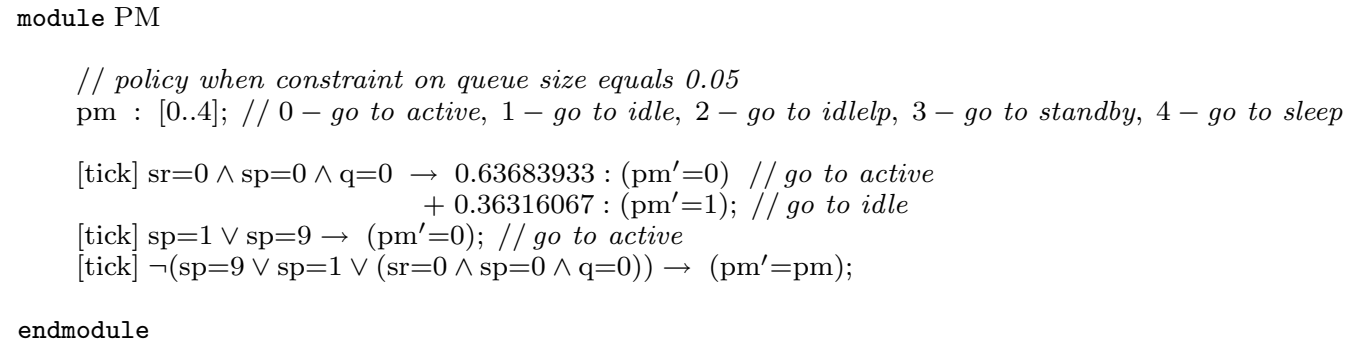

Fig. 9. PRISM module for the power manager under performance constraint 0.05

Table 4. DTMC case study: Power versus performance

\begin{tabular}{cccc}
\hline $\begin{array}{c}\text { policy } \\
\text { constraint }\end{array}$ & $\begin{array}{c}\text { average power } \\
\text { consumption }\end{array}$ & $\begin{array}{c}\text { average queue } \\
\text { size }\end{array}$ & $\begin{array}{c}\text { average number } \\
\text { of lost requests }\end{array}$ \\
\hline 0.001 & 2.460629 & 0.001000 & 0.000106 \\
0.05 & 2.282590 & 0.050000 & 0.000106 \\
0.1 & 2.060040 & 0.100000 & 0.000106 \\
0.2 & 1.670410 & 0.200000 & 0.001671 \\
0.3 & 1.583163 & 0.300000 & 0.011770 \\
0.4 & 1.495917 & 0.400000 & 0.021869 \\
0.5 & 1.408671 & 0.500000 & 0.031968 \\
0.6 & 1.321424 & 0.600000 & 0.042067 \\
0.7 & 1.234178 & 0.700000 & 0.052166 \\
0.8 & 1.146932 & 0.800000 & 0.062265 \\
0.9 & 1.059686 & 0.900000 & 0.072364 \\
1.0 & 0.972439 & 1.000000 & 0.082463 \\
1.1 & 0.885193 & 1.100000 & 0.092562 \\
1.2 & 0.797947 & 1.200000 & 0.102661 \\
1.3 & 0.710700 & 1.300000 & 0.112760 \\
1.4 & 0.623454 & 1.400000 & 0.122859 \\
1.5 & 0.536208 & 1.500000 & 0.132958 \\
1.6 & 0.448962 & 1.600000 & 0.143057 \\
1.7 & 0.361715 & 1.700000 & 0.153156 \\
1.8 & 0.274469 & 1.800000 & 0.163255 \\
1.9 & 0.187223 & 1.900000 & 0.173354 \\
2.0 & 0.100000 & 2.000000 & 0.183450 \\
\hline
\end{tabular}

up until time $T$; the second by computing the instantaneous cost at time $T$. Again, we see that policies which consume less power have larger queue sizes and are more likely to lose requests. Here, though, we can get a much clearer view of how these properties change over time. We see, for example, that the expected queue size at time $T$ initially increases and then decreases. This follows from the fact that the strategies wait for the queue to become full before switching the SP on.

Further properties that we can analyse using probabilistic model checking include:

1. the probability that the queue becomes full before time $T\left(\mathcal{P}=\right.$ ? $\left.\left[\diamond^{\leqslant 2 T} \mathrm{q}=\mathrm{QMAX}\right]\right)$;

2. the probability that a request is served by time $T$, given that it arrived into a certain position in the queue $\left(\mathcal{P}=\right.$ ? $\left[\diamond^{\leqslant 2 T}\right.$ served $\left.]\right)$;

3. the probability that $N$ requests get lost by time $T\left(\mathcal{P}=\right.$ ? $\left.\left[\diamond^{\leqslant 2 T} \operatorname{lost}=N\right]\right)$.

Note that, in the formulae, we use time-bounds of $2 T$, as opposed to $T$, since two steps in the model (a 'tick' followed by a 'tock') correspond to one time-unit in the system. To verify the second and third properties, we must add variables to the PRISM model which record the current position of the request under analysis and to count the number of lost requests (up to a maximum on $N$ ), respectively. In Figure 11 we present these results for a range of policies and for a range of values of $T$. The graphs show that: 

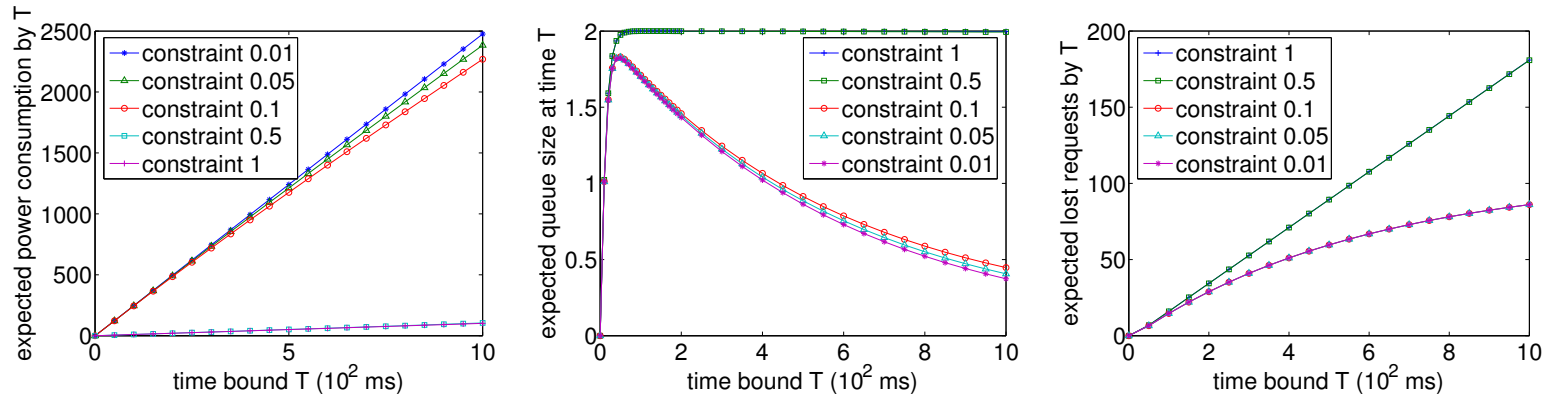

Fig. 10. DTMC case study: power and performance by time $T(\mathrm{~ms})$ for optimal policies under different constraints
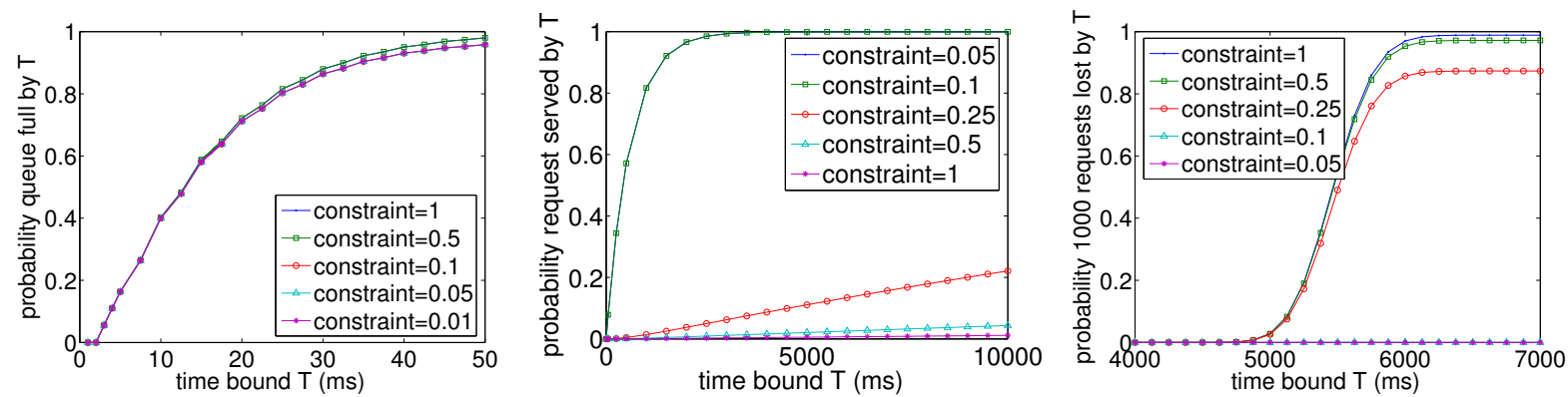

Fig. 11. DTMC case study: analysis of optimal policies for different performance constraints

1. the probability of the queue becoming full within a time bound increases as the constraint on the performance is relaxed (i.e. the requested average queue size is increased);

2. the probability that requests get served increases for policies with stricter performance constraints;

3. the probability of requests being lost within a certain time bound increases more quickly for the strategies with weaker performance constraints.

These results confirm that the policies behave as you would expect. In order to reduce power, the strategies with less strict performance constraints (i.e. with higher values for average queue constraint) can allow the service provider to spend more time in low power states in which service requests cannot be dealt with, e.g. in sleep and standby. For further details on the results obtained, see the PRISM web page [Pri].

\subsection{The Continuous-Time Case}

We have also applied probabilistic model checking to the stochastic optimum control approach of QP99. QWP00, QWP01, which is based on CTMCs rather than DTMCs. The key differences between these two choices of model were given in Section 2. From the point of view of modelling in PRISM, the two are relatively similar. The model has the same basic structure: each component (PM, SP, SRQ and SR) is a separate module and the system is constructed as the parallel composition of these modules. However, in this case we no longer require the CLOCK module to control synchronisation. Since the model is a CTMC, components change state according to exponentially distributed delays and the PM acts when such a state transition occurs.

The construction of optimum policies from the PRISM model now follows the approach of QP99, QWP00, QWP01. We again use MAPLE to perform the solution of optimisation problems for this purpose. For the analysis of policies, we can consider similar properties to the DTMC case. As in the DTMC case, there is a power consumption associated with each power state of the SP (per unit of time); however, there is also a power usage associated with a change in the SP's state. The main differences are that we use the logic CSL as opposed to the logic PCTL, and that the time bound $T$ used in the properties is now a real value as 
opposed to a number of discrete steps. For details on the results obtained using CSL on this case study see the PRISM web page Pri].

Furthermore, in this case, we can also analyse the policies for alternative, more general, inter-arrival time distributions, to give a more realistic model of the arrival of service requests (e.g. Pareto). This can be achieved by modelling the DPM system, for a fixed policy, as a time-homogeneous Markov renewal process Çin75, Kul95 where the renewal point corresponds to the arrival of a new request from the SR. More precisely, we can represent the system as a stochastic process $(X, T)=\left\{X_{n}, T_{n} \mid n \in \mathbb{N}\right\}$, where $X_{n} \in S$ is a random variable corresponding to the state of the system (i.e the state of SP, SR, SRQ and PM) just before the arrival of the $n$th request and $T_{n} \in \mathbb{R}_{\geqslant 0}$ is a random variable corresponding to the time of the arrival of the $n$th request.

For the process $(X, T)$ to be a time-homogeneous Markov renewal process, it must satisfy the following two conditions:

Markovian: for any $n \in \mathbb{N}, s_{1}, \ldots, s_{n+1} \in S$ and $t, t_{1}, \ldots, t_{n} \in \mathbb{R}_{\geqslant 0}$ :

$$
\begin{aligned}
& \mathrm{P}\left(X_{n+1}=s_{n+1}, T_{n+1}-T_{n} \leqslant t \mid X_{0}=s_{0}, \ldots, X_{n}=s_{n} ; T_{1}=t_{1}, \ldots, T_{n}=t_{n}\right) \\
& =\mathrm{P}\left(X_{n+1}=s_{n+1}, T_{n+1}-T_{n} \leqslant t \mid X_{n}=s_{n}\right),
\end{aligned}
$$

i.e. the probability of state change is history-independent.

Time homogeneity: there exists $Q: S \times S \times \mathbb{R}_{\geqslant 0} \rightarrow[0,1]$ such that for any $n \in \mathbb{N}, s, s^{\prime} \in S$ and $t \in \mathbb{R}_{\geqslant 0}$ :

$$
\mathrm{P}\left(X_{n+1}=s^{\prime}, T_{n+1}-T_{n} \leqslant t \mid X_{n}=s\right)=Q\left(s, s^{\prime}, t\right),
$$

i.e. the probability of state change is time-independent.

The satisfaction of (1) and $(2)$ for the process $(X, T)$ considered here follows from the fact that the interarrival time distribution is the only non-exponential distribution in the system (and therefore the only distribution which is non-Markovian) and that the time between the arrival of $n$th and $(n+1)$ th request is given by the inter-arrival time distribution (and is therefore independent of the time between the arrivals of previous requests).

For a time-homogeneous Markov renewal process $(X, T)$, the family $\left\{Q\left(s, s^{\prime}, t\right) \mid s, s^{\prime} \in S, t \in \mathbb{R}_{\geqslant 0}\right\}$ is called a semi-Markov kernel over $S$ and can be used to construct the embedded DTMC $(S, \mathbf{P})$ of the process, where for any $s, s^{\prime} \in S$ :

$$
\mathbf{P}\left(s, s^{\prime}\right)=\lim _{t \rightarrow \infty} Q\left(s, s^{\prime}, t\right) .
$$

More precisely, in the embedded DTMC, the probability of making a transition from state $s$ to $s^{\prime}$ is given by the probability, in the actual process, of being in state $s^{\prime}$ just before the arrival of a request given that the state of the system was $s$ immediately before the arrival of the previous request.

Now, by considering our model as a time-homogeneous Markov renewal process, we can use the theory of Markov renewal processes Çin75, Kul95] to compute performance metrics of the system. For example, under certain restrictions, including that the process is aperiodic and irreducible (which hold for the processes we consider), letting:

$$
\mathbf{C}\left(s, s^{\prime}\right)=\mathrm{E}\left(\text { time spent in state } s^{\prime} \text { during }\left(0, T_{1}\right) \mid X_{0}=s\right)
$$

i.e. the expected time the process spends in state $s^{\prime}$ between two renewal points, given that it started in state $s$ after the last renewal, and letting $\underline{u}$ be the steady state vector of the embedded DTMC, then the limiting state probabilities vector $\underline{\pi}$ of the Markov renewal process are given by:

$$
\underline{\pi}=\frac{\underline{u} \cdot \mathbf{C}}{\underline{u} \cdot \mathbf{C} \cdot \underline{1}} .
$$

The following procedure describes how we have combined probabilistic model checking (in particular the methods given in [KNP02a, KNP02b]) and the theory of Markov renewal processes to analyse the power management policies under general inter-arrival time distributions.

1. Construct a restricted model of the system in which transitions corresponding to new requests are removed. Note that, in this restricted model, all transitions have exponential delay, that is, it is a CTMC.

2. Construct the embedded DTMC of the Markov renewal process described above, that is compute the 
Table 5. CTMC case study: Power versus performance

\begin{tabular}{|c|c|c|c|c|c|c|}
\hline \multirow{2}{*}{$\begin{array}{c}\text { performance } \\
\text { measure }\end{array}$} & \multirow[t]{2}{*}{ constraint } & \multicolumn{5}{|c|}{ inter-arrival time distribution } \\
\hline & & deterministic & exponential & Erlang & uniform & Pareto \\
\hline average & 0.1 & 0.95607 & 0.95732 & 0.95624 & 0.95675 & 0.96085 \\
\hline power & 1 & 0.88649 & 0.89635 & 0.88776 & 0.89168 & 0.93099 \\
\hline consumption & 5 & 0.64712 & 0.63498 & 0.64577 & 0.64265 & 0.43536 \\
\hline average & 0.1 & 0.12454 & 0.10000 & 0.12124 & 0.11101 & 0.028132 \\
\hline queue & 1 & 1.2140 & 1.0000 & 1.1866 & 1.0999 & 0.20238 \\
\hline size & 5 & 5.1573 & 5.0000 & 5.1399 & 5.1021 & 1.6534 \\
\hline average & 0.1 & $2.3677 \mathrm{e}-06$ & $1.1865 \mathrm{e}-05$ & $2.9357 \mathrm{e}-06$ & $4.8316 \mathrm{e}-06$ & $3.5617 \mathrm{e}-03$ \\
\hline number of & 1 & $2.5110 \mathrm{e}-05$ & $1.3217 \mathrm{e}-04$ & $3.1335 \mathrm{e}-05$ & $5.2690 \mathrm{e}-05$ & $4.4060 \mathrm{e}-02$ \\
\hline lost requests & 5 & $1.9618 \mathrm{e}-04$ & $1.0033 \mathrm{e}-03$ & $2.4324 \mathrm{e}-04$ & $4.1157 \mathrm{e}-04$ & $3.3341 \mathrm{e}-01$ \\
\hline
\end{tabular}

probabilities given in $(3)$. These are determined by calculating the probability of satisfying random timebounded until formulae KNP02a on the restricted CTMC model where the random time bound $T$ is set equal to the inter-arrival time distribution of requests. More precisely, for any pair $s, s^{\prime}$ of states, $\mathbf{P}\left(s, s^{\prime}\right)$ is given by the probability, in the restricted CTMC model, of being in state $s^{\prime}$ at the random time $T$, having started in state $\tilde{s}$, where the only difference ${ }^{2}$ between $s$ and $\tilde{s}$ is that in $\tilde{s}$ there is one more request in the queue (SRQ). In the case where the queue is full, $s$ and $\tilde{s}$ are the same. Note that an alternative approach to the construction of the embedded DTMC is to following the methodology of [Ger00].

3. In the restricted CTMC model, calculate, using the techniques developed in KNP02b, the expected reward cumulated until the random time $T$ for the following rewards: time spent in a state, power consumption, queue size and number of lost requests. For example, when the reward is equal to the time spent in state $s^{\prime}$, the expected cumulated reward, starting from state $\tilde{s}$, gives the the values $\mathbf{C}\left(s, s^{\prime}\right)$ of (4), where the difference between $s$ and $\tilde{s}$ is as in step 2 . When the reward is equal to the power consumption, the expected cumulated reward gives the expected power consumption between renewal points.

4. Using the theory of Markov renewal processes, calculate performance and power metrics of the system, through the analysis of the embedded DTMC constructed in step 2 and the expected reward values computed in step 3. For example, the long-run average power consumption can be computed by combining the limiting state probabilities and the expected power consumption between two renewal points of the renewal process. The limiting state probabilities are computed using (5) (i.e. combining the steady state probabilities of the DTMC constructed in step 2 and the values $\mathbf{C}\left(s, s^{\prime}\right)$ computed in step 3$)$ and the expected expected power consumption between two renewal points is calculated in step 3 .

We now present a selection of results to illustrate the utility of the procedure described above. Table 5 shows average power consumption, average queue size and average number of lost requests for optimum policies under five different inter-arrival distributions. The distributions chosen all have the same mean and it can be seen that, with the exception of the Pareto distribution, the long-run performance and costs are reasonably close to those of the exponential arrival process. For the Pareto distribution, the average queue size is generally much smaller. This is a result of the Pareto distribution's heavy tail, which means that, in the long run, many requests will not arrive for a very long time, and hence, in these cases, the service provider (SP) will serve all pending requests, and then the system will spend a long time with the queue empty and the SP in its sleep state consuming very little power. Moreover, more requests are blocked for the Pareto distribution than with the other distributions.

In Figure 12, we present the power and performance results up until the arrival of the $N$ th request as the inter-arrival time distribution varies for the optimum policies for the cases when the constraint on the queue size is 5,1 and 0.1 . Note that, since the distributions we consider have the same mean, the expected arrival time of the $N$ th request is the same for all the distributions considered. Again, we see that the results are very similar in all cases except when the inter-arrival time has a Pareto distribution. Comparing the results we see that, as for the DTMC case study, for larger constraints the power consumption decreases while the expected queue size and expected number of lost requests increases.

\footnotetext{
2 Recall that $X_{n}$ corresponds to the state of the system immediately before the arrival of the $n$th request.
} 

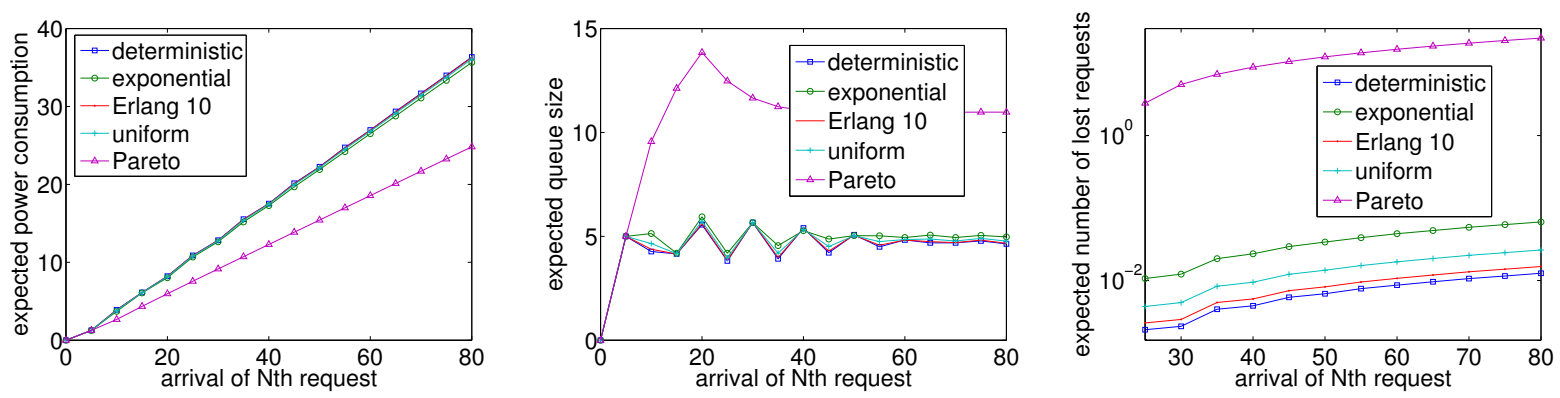

(a) performance constraint of 5
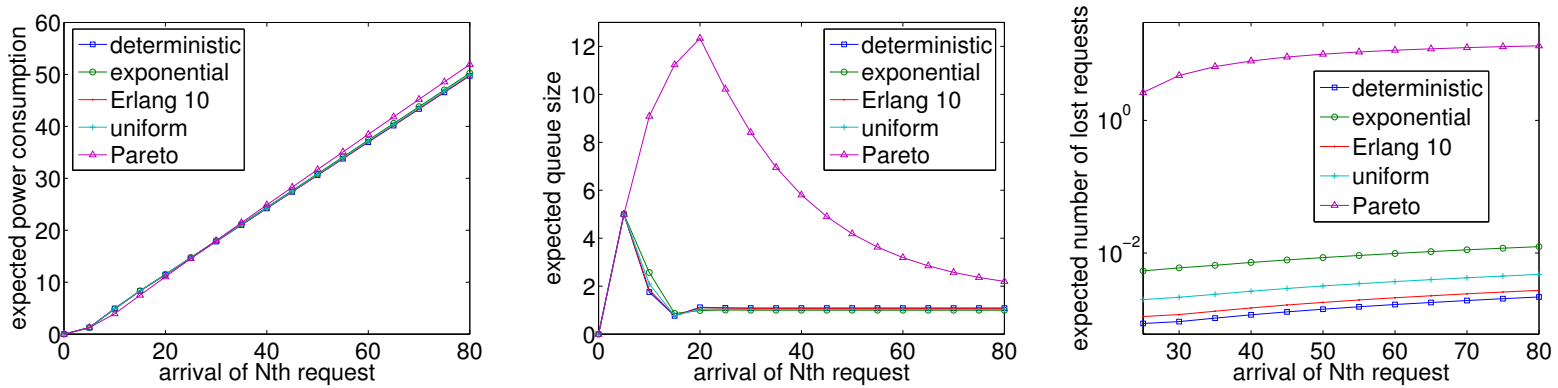

(b) performance constraint of 1
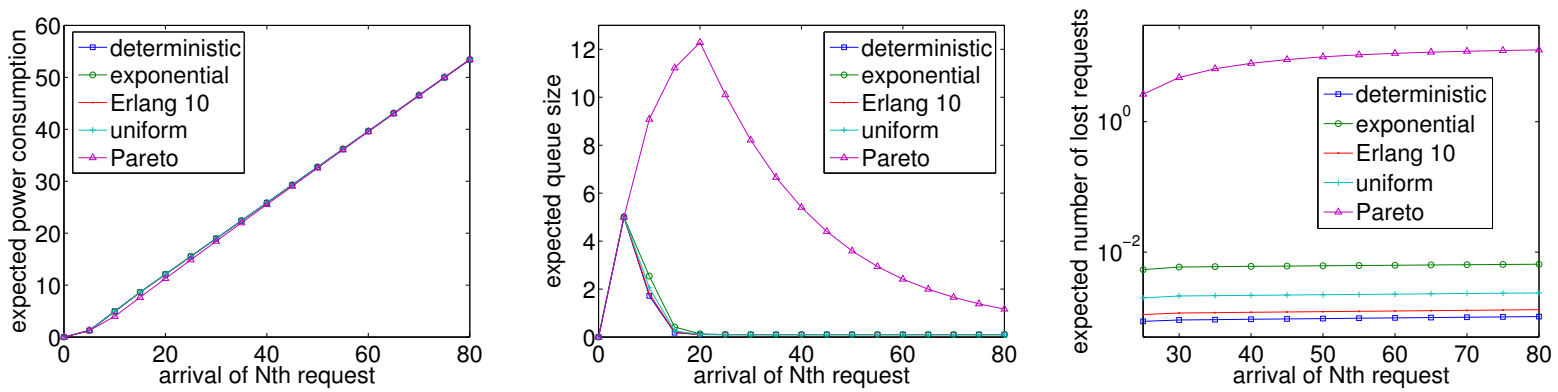

(c) performance constraint of 0.1

Fig. 12. CTMC case study: Power and performance results by the time the $N$ th request arrives

In Figure 13 , we plot the probability that the queue size becomes greater than 10 before the arrival of the $N$ th request and the probability that a request is lost before the arrival of the $N$ th request. The results show that, as the constraint on the performance is relaxed (increased), the probability that there are more than 10 requests waiting in the queue, or that a request is lost before the arrival of the $N$ th request, increases. The rationale is the same as for the DTMC case study: the strategies with low performance constraints (i.e. the higher values on the constraint) can allow the service provider to spend more time in low power states which cannot service requests while still meeting the constraint.

Finally, in all the results, we note the similarity between the cases for requests arriving with a deterministic or Erlang distribution; this is to be expected since the Erlang distribution is often used as a continuous approximation of a (discrete) deterministic distribution [Tri01.

\section{Conclusions}

We have shown that probabilistic model checking allows for the automatic generation of a wide range of performance measures for the analysis of DPM policies. Statistics such as power consumption, service queue 


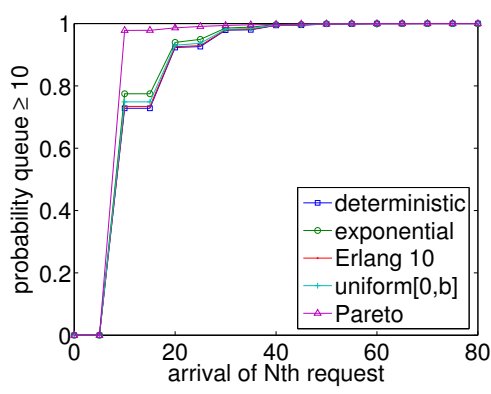

(a) performance constraint of 5

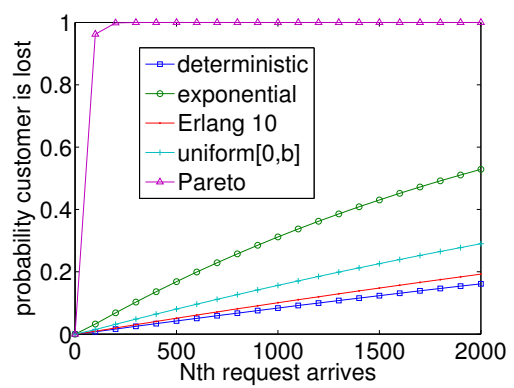

(d) performance constraint of 5

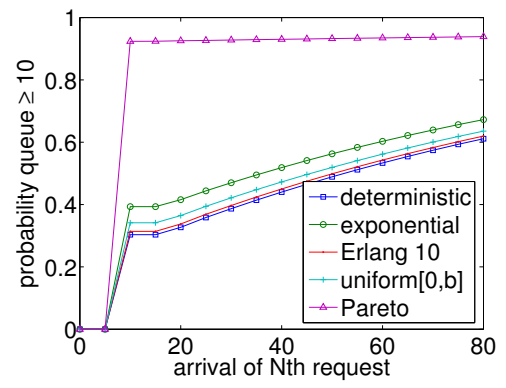

(b) performance constraint of 1

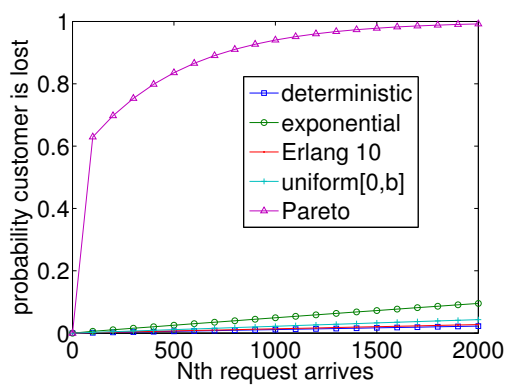

(e) performance constraint of 1

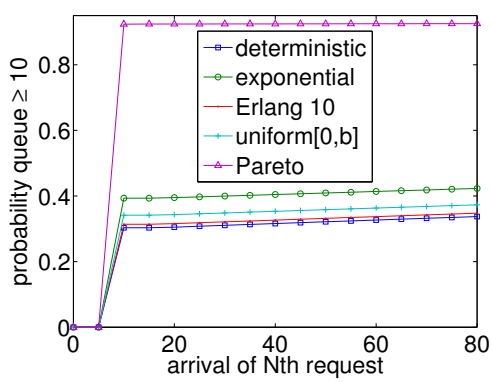

(c) performance constraint of 0.1

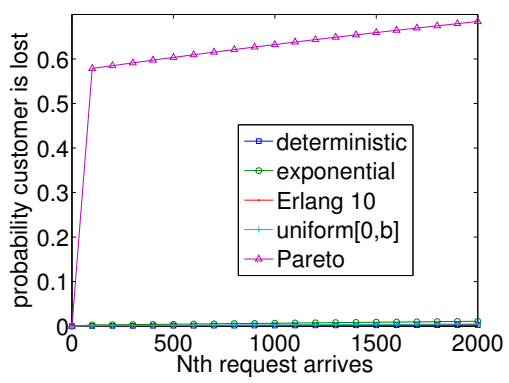

(f) performance constraint of 0.1

Fig. 13. CTMC case study: analysis of optimal policies for different performance constraints

length and the number of requests lost can be computed, both in the average case and for particular time instances over a given range. The fact that a constructed policy is only known to be optimal in the average case makes this information particularly interesting. Furthermore, the policies' behaviour can be examined under alternative, more realistic, service request inter-arrival time distributions such as Erlang and Pareto.

An inherent advantage of the model checking approach is that the analysis of the state-space is exhaustive, in contrast to, say, simulation. This means that the answers computed are guaranteed to be accurate with respect to the probabilistic model used, and that all behaviour, including corner-case scenarios, is considered. We are presently extending this work by building an analytical framework that derives and analyses strategies for more general probabilistic assumptions. We are also considering applying our methodology to DPM schemes with multiple power-managed devices.

\section{Acknowledgements}

This work was supported by NSF grants CCR-0098335 and CCR-0340740, EPSRC grants GR/N22960 and GR/S11107, FORWARD, SRC, and DARPA/ITO supported PADS project under the PAC/C program.

\section{References}

[ACP $] \quad$ Advanced configuration and power interface website (www.acpi.info).

[AH99] R. Alur and T. Henzinger. Reactive modules. Formal Methods in System Design, 15:7-48, 1999.

[ASSB96] A. Aziz, K. Sanwal, V. Singhal, and R. Brayton. Verifying continuous time Markov chains. In R. Alur and T. Henzinger, editors, Proc. 8th Int. Conf. Computer Aided Verification (CAV'96), volume 1102 of Lecture Notes in Computer Science, pages 269-276. Springer-Verlag, 1996.

[BBM00] L. Benini, A Bogliolo, and G. De Micheli. A survey of design techniques for system-leveldynamic power management. IEEE Transactions on Very Large Scale Integration (TVLSI) Systems, 8(3):299-316, 2000.

[BBPM99] L. Benini, A Bogliolo, G. Paleologo, and G. De Micheli. Policy optimization for dynamic power management. IEEE Transactions on Computer-Aided Design of Integrated Circuits and Systems, 18(6):813-833, 1999.

[BdA95] A. Bianco and L. de Alfaro. Model checking of probabilistic and nondeterministic systems. In P. Thiagarajan, 
editor, Proc. 15th Conf. Foundations of Software Technology and Theoretical Computer Science, volume 1026 of Lecture Notes in Computer Science, pages 499-513. Springer-Verlag, 1995.

[Ber95] D. Bertsekas. Dynamic Programming and Optimal Control, Volumes 1 and 2. Athena Scientific, 1995.

[BHHK00] C. Baier, B. Haverkort, H. Hermanns, and J.-P. Katoen. On the logical characterisation of performability properties. In U. Montanari, J. Rolim, and E. Welzl, editors, Proc. 27th Int. Colloquium on Automata, Languages and Programming (ICALP'O0), volume 1853 of Lecture Notes in Computer Science, pages 780-792. Springer-Verlag, 2000.

[BKH99] C. Baier, J.-P. Katoen, and H. Hermanns. Approximate symbolic model checking of continuous-time Markov chains. In J. Baeten and S. Mauw, editors, Proc. 10th Int. Conf. Concurrency Theory (CONCUR'99), volume 1664 of Lecture Notes in Computer Science, pages 146-161. Springer-Verlag, 1999.

[BM98] L. Benini and G. De Micheli. Dynamic Power Management: Design Techniques and CAD Tools. Kluwer Publications, 1998.

[BMM01] L. Benini, G. De Micheli, and E. Macii. Designing low-power circuits: Practical recipes. IEEE Circuits and Systems Magazine, 1(1):6-25, 2001.

[BT91] D. Bertsekas and J. Tsitsiklis. An analysis of stochastic shortest path problems. Mathematics of Operations Research, 16(3):580-595, 1991.

[CBBM99] E.-Y. Chung, L. Benini, A. Bogliolo, and G. De Micheli. Dynamic power management for non-stationary service requests. In Proc. Design, Automation and Test in Europe (DATE'99), pages 77-81. IEEE Computer Society Press, 1999.

[Cin75] E. Cinlar. Introduction to Stochastic Processes. Prentice Hall, 1975.

[dA97] L. de Alfaro. Formal Verification of Probabilistic Systems. PhD thesis, Stanford University, 1997.

[Ger00] R. German. Performance Analysis of Communication Systems: Modeling with Non-Markovian Stochastic Petri Nets. John Wiley and Sons, 2000.

[HAW96] C.-H. Hwang, C. Allen, and H. Wu. A predictive system shutdown method for energy saving of event-driven computation. In Proc. Int. Conf. on Computer-Aided Design (ICCAD'97), pages 28-32. IEEE Computer Society Press, 1996.

[HGCC99] V. Hartonas-Garmhausen, S. Campos, and E. Clarke. ProbVerus: Probabilistic symbolic model checking. In J.-P. Katoen, editor, Proc. 5th Int. AMAST Workshop on Real-Time and Probabilistic Systems (ARTS'99), volume 1601 of Lecture Notes in Computer Science, pages 96-110. Springer-Verlag, 1999.

[HJ94] H. Hansson and B. Jonsson. A logic for reasoning about time and probability. Formal Aspects of Computing, 6:512-535, 1994.

[HKMKS00] H. Hermanns, J.-P. Katoen, J. Meyer-Kayser, and M. Siegle. A Markov chain model checker. In S. Graf and M. Schwartzbach, editors, Proc. 6th Int. Conf. Tools and Algorithms for the Construction and Analysis of Systems (TACAS 2000), volume 1785 of Lecture Notes in Computer Science, pages 347-362. Springer-Verlag, 2000.

[IBM] Technical specifications of hard drive IBM Travelstar VP (www.storage.ibm.com/storage/oem/data/travvp.htm).

[ISG02] S. Irani, S. Shukla, and R. Gupta. Competitive analysis of dynamic power management strategies for systems with multiple power saving states. In Proc. Design Automation and Test Conference (DATE 2002), pages 117-123. IEEE Computer Society Press, 2002.

[JDL02] B. Jeannet, P. D'Argenio, and K. Larsen. RAPTURE: A tool for verifying Markov decision processes. In I. Cerna, editor, Proc. Tools Day, affiliated to 13th Int. Conf. Concurrency Theory (CONCUR'02), Technical Report FIMURS-2002-05, Faculty of Informatics, Masaryk University, pages 84-98, 2002.

[KNP02a] M. Kwiatkowska, G. Norman, and A. Pacheco. Model checking CSL until formulae with random time bounds. In H. Hermanns and R. Segala, editors, Proc. 2nd Joint Int. Workshop on Process Algebra and Probabilistic Methods, Performance Modeling and Verification (PAPM/PROBMIV'02), volume 2399 of Lecture Notes in Computer Science, pages 152-168. Springer-Verlag, 2002.

[KNP02b] M. Kwiatkowska, G. Norman, and A. Pacheco. Model checking expected time and expected reward formulae with random time bounds. In Proc. 2nd Euro-Japanese Workshop on Stochastic Risk Modelling for Finance, Insurance, Production and Reliability, 2002.

[KNP04] M. Kwiatkowska, G. Norman, and D. Parker. PRISM 2.0: A tool for probabilistic model checking. In Proc. 1st International Conference on Quantitative Evaluation of Systems (QEST'04), pages 322-323. IEEE Computer Society Press, 2004.

[Kul95] V. Kulkarni. Modeling and Analysis of Stochastic Systems. Chapman and Hall, 1995.

[Map] MapleSoft Corporation. Maple computer algebra system (www.maplesoft.com).

[Mic98] Microsoft corporation, OnNow power management architecture for applications (msdn.microsoft.com/library/enus/dndevio/html/onnowapp.asp). 1998.

$\left[\mathrm{NPK}^{+} 02\right]$ G. Norman, D. Parker, M. Kwiatkowska, S. Shukla, and R. Gupta. Formal analysis and validation of continuous time Markov chain based system level power management strategies. In W. Rosenstiel, editor, Proc. 7th Annual IEEE Int. Workshop on High Level Design Validation and Test (HLDVT'02), pages 45-50. IEEE Computer Society Press, 2002.

$\left[\mathrm{NPK}^{+} 03\right]$ G. Norman, D. Parker, M. Kwiatkowska, S. Shukla, and R. Gupta. Using probabilistic model checking for dynamic power management. In M. Leuschel, S. Gruner, and S. Lo Presti, editors, Proc. 3rd Workshop on Automated Verification of Critical Systems (AVoCS'03), Technical Report DSSE-TR-2003-2, University of Southampton, pages 202-215, 2003.

[Par02] D. Parker. Implementation of Symbolic Model Checking for Probabilistic Systems. PhD thesis, University of Birmingham, 2002.

[PBBM98] G. Paleologo, L. Benini, A. Bogliolo, and G. Micheli. Policy optimization for dynamic power management. In Proc. 35th Conf. Design Automation (DAC 1998), pages 182-187. ACM Press, 1998. 
[Pri] PRISM web page (www.cs.bham.ac.uk/ dxp/prism).

[QP99] Q. Qiu and M. Pedram. Dynamic power management based on continuous-time Markov decision processes. In Proc. 36th Conf. Design Automation (DAC 1999), pages 555-561, 1999

[QWP00] Q. Qiu, Q. Wu, and M. Pedram. Dynamic power management of complex systems using generalized stochastic Petri nets. In Proc. 37th Conf. Design Automation (DAC 2000), pages 352-356, 2000.

[QWP01] Q. Qiu, Q. Wu, and M. Pedram. Stochastic modeling of a power-managed system: construction and optimization. IEEE Transactions on Computer Aided Design, 20(9):1200-1217, 2001.

[RIG00] D. Ramanathan, S. Irani, and R. Gupta. Latency effects of system level power management algorithms. In Proc. IEEE Int. Conf. Computer-Aided Design, 2000.

[SBM99] T. Simunic, L. Benini, and G. De Micheli. Event driven power management of portable systems. In Proc. Int. Symp. System Synthesis (ISSS 1999), pages 18-23. IEEE Computer Society Press, 1999.

[SCB96] M. Srivastava, A. Chandrakasan, and R. Broderson. Predictive shutdown and other architectural techniques for energy efficient programmable computation. IEEE Trans. on VLSI Systems, 4(1):42-54, 1996.

[SG01] S. Shukla and R. Gupta. A model checking approach to evaluating system level power management for embedded systems. In Proc. IEEE Workshop on High Level Design Validation and Test (HLDVT'01), pages 53-57. IEEE Computer Society Press, 2001.

[Tri01] K. Trivedi. Probability and Statistics with Reliability, Queuing, and Computer Science Applications. John Wiley and Sons, 2001. 